\title{
Bilateral Paragangliomas in the Setting of Autonomic Dysfunction: A Case Report
}

\author{
Jerry Shen ${ }^{a}$ Angela Ryck ${ }^{a}$ Iris Chan ${ }^{a}$ Kaitlin S. McFadden ${ }^{b}$ \\ Anna D. Hohler ${ }^{\mathrm{C}}$ \\ aSchool of Medicine, Tufts University School of Medicine, Boston, MA, USA; beuroscience, \\ Mount Holyoke College, South Hadley, MA, USA; 'Department of Neurology, St. Elizabeth's \\ Medical Center, Boston, MA, USA
}

\section{Keywords}

Paraganglioma · Hypertension · Carotid body · Autonomic dysfunction · Case report

\begin{abstract}
In 2018, a 59-year-old female patient presented with hoarseness in her voice, headache, intermittent pain in her right side, difficulty of right arm movement, left side neck pain, difficulty controlling hypertension of unknown etiology, and a large mass on the upper left side of her neck with a smaller mass on the right side. MRI of the neck revealed masses at each carotid bifurcation. These were determined to be bilateral paragangliomas. Paragangliomas are rare tumors, and bilateral ones tremendously so. The patient underwent radiation over 2 years, resulting in the successful shrinking and stabilization of both masses. Since completing radiation, the patient reported improvement in her memory, and her blood pressure has stabilized with medication.
\end{abstract}

(C) 2021 The Author(s).

Published by S. Karger AG, Basel

\section{Introduction}

Paragangliomas are rare neuroendocrine tumors that typically arise from structures associated with the central nervous system and the extra-adrenal autonomic paraganglia. They are often slow growing, and the vast majority are benign [1]. Paragangliomas can be divided into 2 groups: sympathetic and parasympathetic tumors. Nearly all head and neck 
paragangliomas arise from the parasympathetic nervous system. These parasympathetic paragangliomas can be further described by the site of origin, including the carotid, jugular, or vagal. Patients with clinical signs or symptoms indicating excessive catecholamine release are evaluated for the presence of a functional paraganglioma or multiple tumors. Multiple paragangliomas can occur in up to $40 \%$ of familial paragangliomas and in up to $10 \%$ of sporadic tumors [2]. The rarity of bilateral malignant paragangliomas makes their management clinically challenging. If paragangliomas are suspected, MRI is the modality of choice, and the tumors can be characterized by their classic appearance and relation to surrounding structures.

Recent molecular studies have shown that some paragangliomas have been germline mutations, and 6 genes have been identified including RET, VHL, NF1, and succinate dehydrogenase (SDH). SDH subunit mutations are very important for head and neck paragangliomas. Paragangliomas may be hereditary and may be a part of genetic syndromes such as multiple endocrine neoplasia (MEN) 2A and MEN 2B, Von Hippel-Lindau syndrome, and neurofibromatosis type 1. Although many familial cases that are often associated with the abovementioned germline mutations go unrecognized, germline mutations have been reported in $7.5 \%-24 \%$ [3].

While most reported cases are benign and unilateral, we present here a rare case of bilateral paragangliomas with autonomic dysfunction. This is an important case as it is a rare condition when occurring bilaterally that is relatively easily diagnosed by a good history and clinical examination, using MRI scanning as an aid to diagnosis and follow-up.

\section{Case Report}

The patient was a 59-year-old woman with a history of hypertension and progressive short-term memory issues who initially presented for evaluation of a mass on the left side of her neck that she noted 3 years prior to presentation, which has grown progressively larger. She also reported hoarseness, a feeling of a lump in her throat, and occasional nonproductive cough over the last month. The patient also has a family history of an unknown clotting disorder and stroke. Of note, prior to initial presentation, the patient had experienced difficulty with controlling her blood pressure on medications for several years. She reported that her systolic pressure could suddenly increase as high as $217 \mathrm{~mm} \mathrm{Hg}$, and she endorsed experiencing headaches, dizziness, and difficulties focusing during these episodes of higher blood pressure.

Upon physical examination, easily palpable, slightly mobile, and nontender masses were noted bilaterally in the anterior upper neck. MRI of the neck revealed soft tissue masses enhancing at both carotid bifurcations, found to be bilateral carotid body paragangliomas, shown in Figure 1. The right neck mass measured $2.9 \mathrm{~cm}$ in the greatest craniocaudal dimension, and the left neck mass measured $2.6 \mathrm{~cm}$ in the greatest craniocaudal dimension, shown in Figure 2.

The patient underwent 10 rounds of radiation therapy over 2 years, resulting in shrinking of both paragangliomas. The masses have been stable, and there has been no plan for additional radiation therapy. Since finishing radiation therapy, the patient has expressed an improvement in her memory and voice, and her blood pressure has been better controlled on amlodipine and hydrochlorothiazide. It remains unclear whether her hypertension is secondary to compression of the carotid arteries or direct carotid body stimulation.

\section{Discussion}

The significance of this case lies in the fact that paragangliomas are uncommon neuroendocrine tumors, and multiple paragangliomas in 1 patient are exceedingly rare. Only $3 \%$ of paragangliomas occur in the head and neck region [4]. Paragangliomas that occur bilaterally

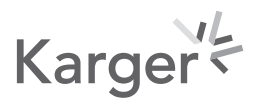




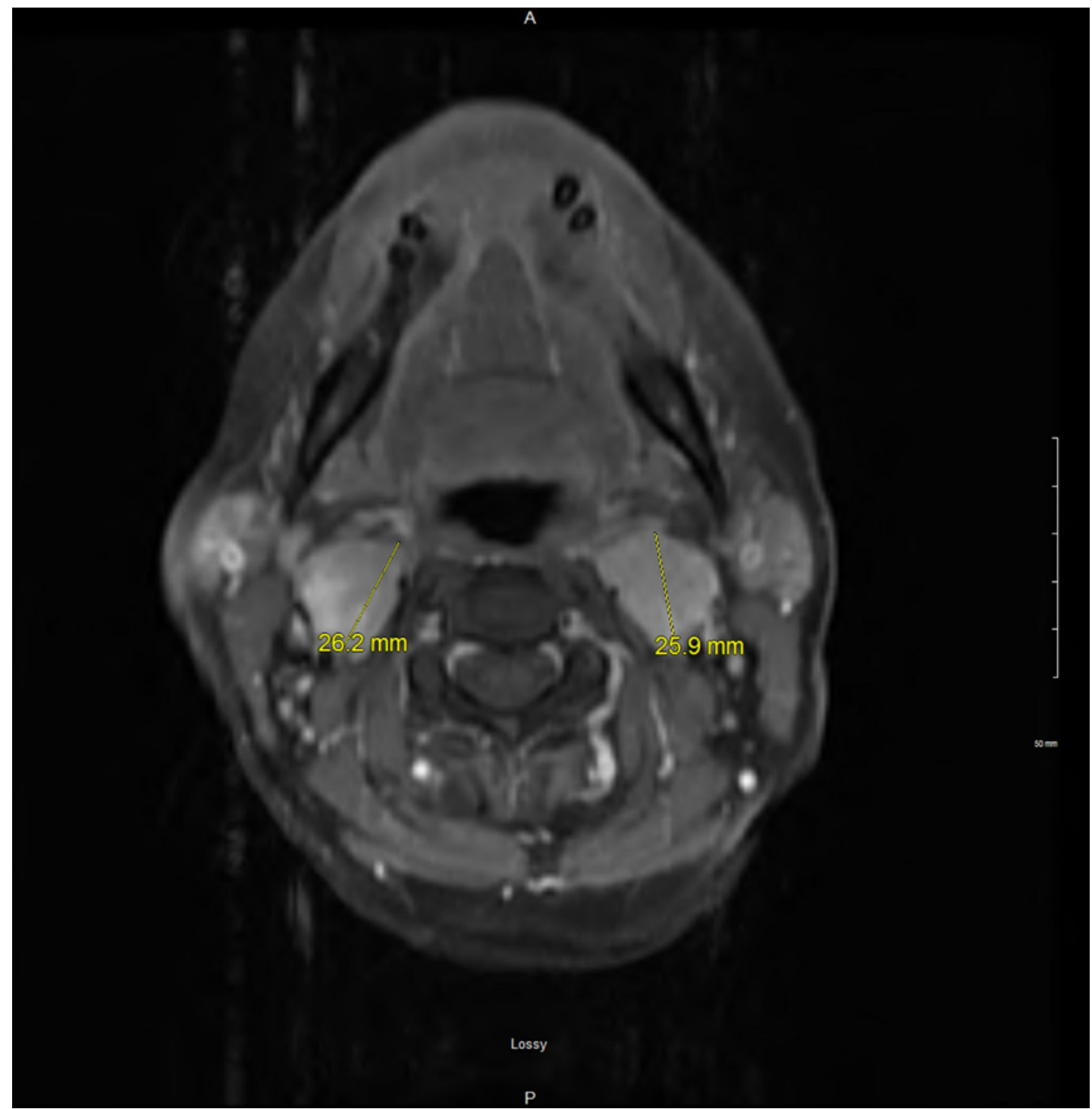

Fig. 1. Axial section of noncontrast magnetic resonance imaging of the orbit, neck, and face reveals bilateral paragangliomas at both carotid bifurcations.

are more likely to be of familial origin than sporadic [5]. Previous studies have noted an association between familial paragangliomas and a germline mutation of succinate dehydrogenase subunits SDHD on chromosome 11q23 [4]. While familial origin tends to be more common in bilateral paragangliomas, this does not appear to be the case in the patient described in this case. She denies having family members with paragangliomas, so genetic testing has not been pursued at this time. However, she does note a clotting disorder in several close relatives.

In reviewing the literature, patients often note the presence of neck masses for years before their diagnosis, supporting the fact that these are slow-growing tumors [6]. Paragangliomas are generally benign tumors, and only rarely become malignantand metastasize [5]. Surgery is generally considered the primary definitive treatment for paragangliomas [7]. Surgical removal has been utilized for several cases of bilateral paragangliomas [8, 9]. However, due to the locations of this patient's tumors on such critical vessels, she underwent targeted radiation therapy to reduce blood flow to the tumors. The patient noted improvement of her symptoms as she progressed through 


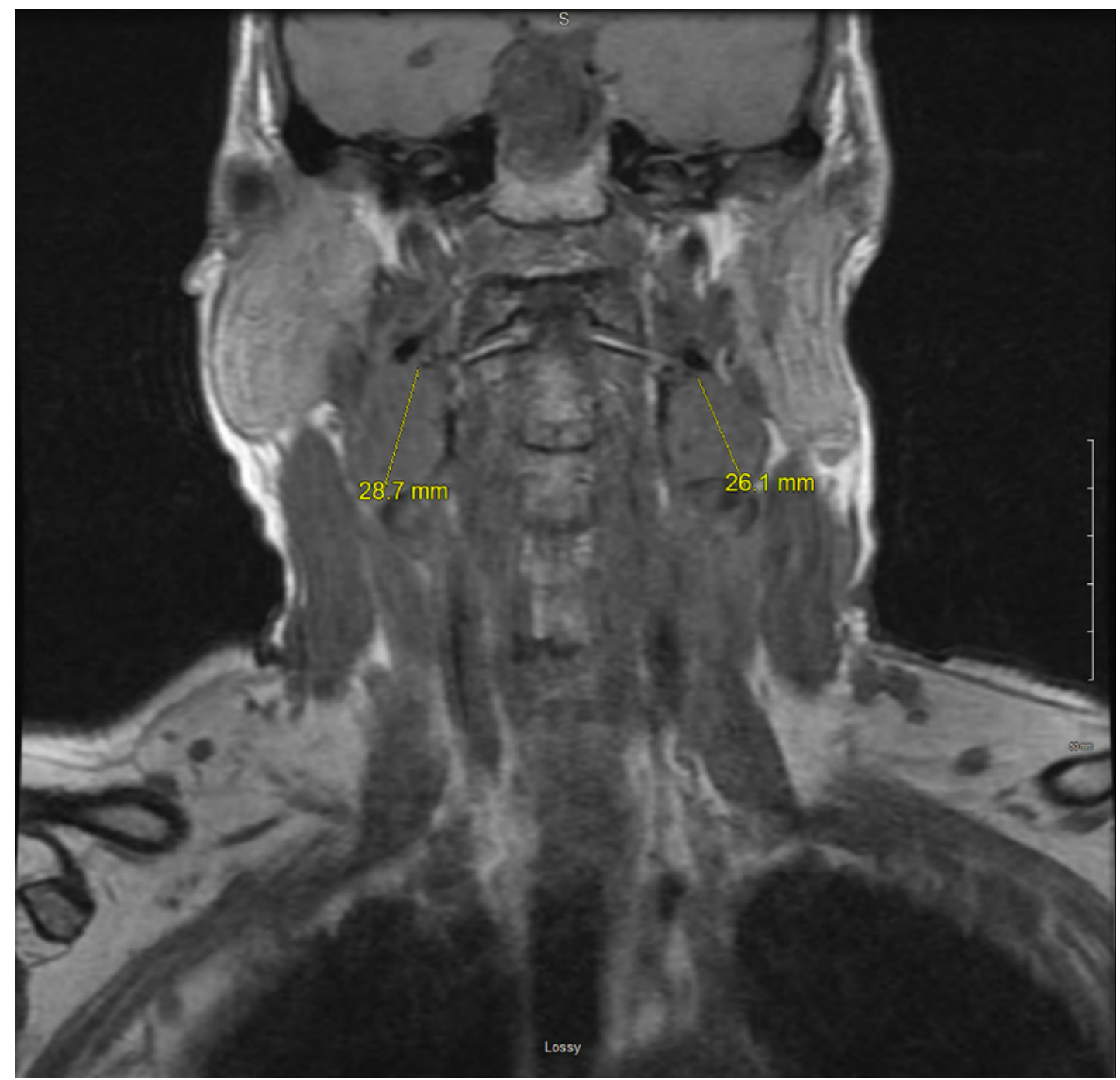

Fig. 2. Coronal section of noncontrast magnetic resonance imaging of the orbit, neck, and face reveals bilateral paragangliomas at both carotid bifurcations.

radiation therapy. Interestingly, she describes testing her progress by comparing the sound and feel of her singing voice, noting a more normal and fluid voice as she improved with treatment. A 2019 case report by Lin et al. [4] describes a patient who similarly experienced resolution of symptoms after undergoing radiation treatment for bilateral paragangliomas.

The patient we present described feeling uncertain and worried about what she can expect with this rare diagnosis. She also noted stress about the potential implications of the diagnosis from a genetic perspective and what that could mean for her children. Last, the patient explained that the process of radiation therapy was time consuming and physically uncomfortable. Providers can take steps to ensure patients are well informed and cared for during their course of care. Upon being diagnosed with this type of tumor, patients should be advised that this is a very slowly-growing tumor that is unlikely to be malignant. Patients should understand that while surgical treatment is ideal, radiation therapy is often effective at both stabilizing or reducing tumor size and reducing or resolving symptoms. Optimization of management and patient education can ensure a successful outcome after diagnosis with paragangliomas, even those producing autonomic dysfunction due to carotid compression. 


\section{Statement of Ethics}

This study protocol was reviewed and approved by St. Elizabeth's Medical Center, Approval No. NHSR005. The study was exempt from a full IRB review. Written informed consent was obtained from the participant for publication of the details of the medical case and any accompanying images.

\section{Conflict of Interest Statement}

The authors have no conflicts of interest to declare.

\section{Funding Sources}

There are no sources of funding to report.

\section{Author Contributions}

J.S. contributed to the writing of the case presentation section, analysis of the patient interview, figures of the manuscript, and the cover letter. A.R. coordinated the patient interview, contributed to the analysis of the patient interview, and contributed to the writing of the Discussion section. I.C. contributed to the analysis of the patient interview and figures, writing of the Introduction section, and editing of the Discussion section. K.S.M. contributed to the analysis of the patient interview and writing of the abstract. All authors reviewed and approved the final version of the manuscript.

\section{Data Availability Statement}

All data generated or analyzed during this study are included in this article. Further inquiries can be directed to the corresponding author.

\section{References}

1 Trivedi RY, Leong SC. Bilateral paraganglioma. BMJ Case Rep. 2012 Nov 9;2012:2012PMC4542629.

2 Kimura NCR, Capella C, Young W, Chetty R, Koch CA, Lam A, et al. Extra-adrenal paraganglioma: carotid body, jugulotympanic, vagal, laryngeal, aortico-pulmonary. In: DeLellis RA, Heitz PU, Eng C, editors. World health organization classification of tumours pathology and genetics: tumours of endocrine organs. Lyon, France: IARC Press; 2004. p. 159-61.

3 Fishbein L, Nathanson KL. Pheochromocytoma and paraganglioma: understanding the complexities of the genetic background. Cancer GenetPMCID. 2012 Jan;205(1-2):1PMC3311650-11.

4 Lin B, Yang H, Yang H, Shen S. Bilateral malignant paragangliomas in a patient: a rare case report. World Neurosurg. 2019 Jan 3(18):S187832954-87501.

5 Ikram A, Rehman A. StatPearls [Internet]. Treasure Island, FL: StatPearls Publishing; 2021.

6 Amith S, Rajesh BS, Dutta S, Anandhi A. Sporadic bilateral carotid body paragangliomas. BMJ Case Rep. 2020 Aug 24;13(8):e237668.

7 Aygun N, Uludag M. Pheochromocytoma and paraganglioma: from treatment to follow-up. Sisli Etfal Hastan Tip Bul. 2020 Dec 11;54(4):391-8.

8 Wang SH, Chiu KM, Cheng PW. Bilateral carotid body paragangliomas. CMAJPMCID. 2011 Jun 14;183(9): E606PMC3114940.

9 Mumoli N, Cei M, Pauletti M, Ferrito G, Scazzeri F. Bilateral neck paragangliomas. QJM. October 2009;102(10):733-5.

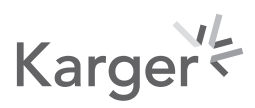

